\title{
Irrotational Binary Neutron Stars in Quasi-Equilibrium
}

\author{
P. Marronetti and G. J. Mathews \\ University of Notre Dame, Department of Physics, Notre Dame, Indiana 46556 \\ J. R. Wilson \\ University of California, Lawrence Livermore National Laboratory, Livermore, California 94550
}

(September 28, 2018)

We report on numerical results from an independent formalism to describe the quasi-equilibrium structure of nonsynchronous binary neutron stars in general relativity. This is an important independent test of controversial numerical hydrodynamic simulations which suggested that nonsynchronous neutron stars in a close binary can experience compression prior to the last stable circular orbit. We show that, for compact enough stars the interior density increases slightly as irrotational binary neutron stars approach their last orbits. The magnitude of the effect, however, is much smaller than that reported in previous hydrodynamic simulations.

PACS number(s): 97.80.Fk, 04.25.Dm, 04.40.Dg, 97.60.Jd

\section{INTRODUCTION}

The physical processes occurring during the last orbits of a neutron-star binary are a subject of intense current debate 11 11]. In part, this recent surge in interest stems from relativistic numerical hydrodynamic simulations in which it has been noted [1] 3] that as the stars approach each other their interior density increases. Indeed, for an appropriate mass and equation of state, previous numerical simulations indicated that binary neutron stars might collapse individually toward black holes seconds prior to merger. This compression effect would have a significant impact on the anticipated gravity-wave signal from merging neutron stars and may also provide an energy source for cosmological gamma-ray bursts [3].

In view of the unexpected nature of this neutron star compression effect, and its possible repercussions, as well as the extreme complexity of strong field general relativistic hydrodynamics, it is of course imperative that there be an independent confirmation of the existence of neutron star compression before one can be convinced of its operation in binary systems. This is particularly important since many authors [5 10] have searched for but not observed this effect in Newtonian tidal forces [5], first post-Newtonian (1PN) dynamics [6, 10], tidal expansions [7,8], or in binaries in which rigid corotation has been imposed [9,12]. In ref. [4] it has been argued that none of the above works could or should have observed the effect, since the compression only dominates over tidal forces for stars with a realistic compaction ratio $\left[\left(M_{G} / R\right)_{\infty}\right]$ that are in a nearly irrotational hydrodynamic state (i.e. little spin relative to a distant observer). Indeed, irrotational stars may be a likely configuration near the final orbits as corotation would demand an unrealistically large viscosity in neutron stars 13 . In hydrodynamic relaxation calculations [4 the stars even seem to prefer a nearly irrotational state. Rasio and Shapiro [14 provide a recent review of the state of the aforementioned controversy.

With this in mind it is of particular interest that a new formalism [15 19] has been proposed in which the hydrostatic quasi-equilibrium of irrotational stars in a binary can be solved independently of the complexities of $(3+1)$ numerical relativistic hydrodynamics. Thus, this provides an opportunity to independently test the hydrodynamics result. Previous results using this formalism have been presented by Bonazzola et al. [11]. They show that for an irrotational binary system composed of neutron stars with $\left(M_{G} / R\right)_{\infty}$ of 0.14 there is almost no evidence of the compression effect (although they did note that the central density for irrotational stars remains much higher than for corotating stars as the orbit decays). In this paper we report results for two different systems involving stars with $\left(M_{G} / R\right)_{\infty}$ of 0.14 and 0.19 . We show that, while the former sequence shows almost no compression in agreement with [11], stars with a higher compaction ratio seem to experience a slight central compression as the stars approach.

We also note that these sequences have an important intrinsic value beyond the controversy about the compression effect. They provide realistic solutions to the initial value problem for neutron-star binary systems that can be used as starting points of fully dynamical relativistic hydrodynamical simulations. The fact that they are valid in the strong field regime makes them more attractive than post-Newtonian counterparts, since the simulations can be initiated at stages very close to the final merger of the stars.

\section{THE METHOD}

The method we use to determine the internal structure of stars in irrotational quasi-equilibrium configurations is essentially that originally proposed by Bonazzola, Gourgoulhon \& Marck [15] and as simplified by Teukolsky [16]. The derivation of the relevant equations can be found in those papers. The essential ingredient of this approach is 
that, if the fluid vorticity is zero (e.g. as in irrotational stars), the specific momentum density per baryon can be written as the gradient of a scalar potential,

$$
h u_{\mu}=\nabla_{\mu} \psi
$$

where $u_{\mu}$ is the covariant four velocity and $h$ is the relativistic enthalpy, $h=1+\epsilon+\frac{P}{\rho_{0}}$, where $\epsilon$ is the internal energy per unit of baryon rest mass, $P$ is the pressure, and $\rho_{0}=m_{B} n_{B}$ is the proper baryon rest mass density, with $n_{B}$ the baryon number density. The potential $\psi$ can be obtained from the solution of a Poisson-like equation:

$$
D^{i} D_{i} \psi=D_{i} \frac{\lambda B^{i}}{\alpha^{2}}-\left(D^{i} \psi-\frac{\lambda}{\alpha^{2}} B^{i}\right) D_{i} \ln \left(\frac{\alpha n_{B}}{h}\right)
$$

where $D_{i}$ are spatial covariant derivatives, and

$$
\lambda=C+B^{j} D_{j} \psi=\alpha\left[h^{2}+\left(D_{i} \psi\right)^{2}\right]^{1 / 2},
$$

where $C$ is a constant. The quantities $\alpha$ and $B^{i}$ are obtained from the usual ADM $(3+1)$ metric

$$
d s^{2}=-\left(\alpha^{2}-\beta_{i} \beta^{i}\right) d t^{2}+2 \beta_{i} d x^{i} d t+\gamma_{i j} d x^{i} d x^{j},
$$

such the $B^{i}$ is the shift vector in the rotating frame, $B^{i}=$ $\beta^{i}+(\omega \times r)^{i}$, where $\omega$ is the angular velocity of orbital motion.

Equation (2) must be solved by imposing a boundary condition at the stellar surface,

$$
\left.\left(D^{i} \psi-\frac{\lambda}{\alpha^{2}} B^{i}\right) D_{i} n_{B}\right|_{\text {surf }}=0 .
$$

For irrotational stars the Bernoulli integral for the matter distribution then becomes:

$$
h^{2}=-\left(D^{i} \psi\right) D_{i} \psi+\frac{\lambda^{2}}{\alpha^{2}} .
$$

Equation (6) uniquely determines the equilibrium structure of the stars.

We also compute stars in constant corotation. In this case the relativistic Bernoulli equation can be written [9, 12] $h / u^{0}=$ constant.

In the present work we consider a polytropic equation of state, $P=K \rho_{0}^{\Gamma}$, with $\Gamma=2$ and $K=1.13 \times 10^{5} \mathrm{erg}$ $\mathrm{cm}^{3} \mathrm{~g}^{-2}$. This gives a maximum neutron-star gravitational mass of $1.46 M_{\odot}$. In these simulations we consider two equal-mass neutron stars in two different sequences of stable orbits. The first corresponds to stars with baryon mass of $M_{B}=1.29 M_{\odot}$, gravitational mass in isolation of $M_{G \infty}=1.21 M_{\odot}$, and compaction ratio of $\left(M_{G} / R\right)_{\infty}=0.14$. This sequence is comparable to the one studied by Bonazzola et al. [11. The second sequence corresponds to a systems of more compact stars, with baryon mass of $M_{B}=1.55 M_{\odot}$, gravitational mass in isolation of $M_{G \infty}=1.41 M_{\odot}$, and compaction ratio of 0.19 . For the grid resolution of this study $(\sim 40$ zones across the star) we obtain a proper central density in isolation of $\rho_{\infty}=0.96 \times 10^{14} \mathrm{~g} \mathrm{~cm}^{-3}$ for the first sequence and $\rho_{\infty}=1.83 \times 10^{14} \mathrm{~g} \mathrm{~cm}^{-3}$ for the second one. As pointed out in [4] it is important to study realistically compact neutron stars. Otherwise Newtonian tidal forces can dominate over the relativistic effects one desires to probe.

The Einstein field equations are solved by imposing a conformally flat condition (CFC) on the three metric [1,2]. That is, the spatial three metric is constrained to be represented by a position dependent conformal factor $\phi^{4}$ times the Kronecker delta, $\gamma_{i j}=\phi^{4} \delta_{i j}$. This is a common metric choice for solving the initial value problem in numerical relativity. It is consistent with the approximation of quasi-equilibrium circular orbits [4, 16] which we wish to evaluate (see ref. [4 for a detailed discussion).

The advantage of this method is that the determination of the metric coefficients reduces to the solution of flat-space Poisson like equations [2]. For example, using the Hamiltonian constraint 20] in combination with the maximal slicing condition $\operatorname{tr}(K)=0$, the equation for $\phi$ becomes

$$
\nabla^{2} \phi=-4 \pi \frac{\phi^{5}}{2}\left[\rho_{0} h W^{2}-P+\frac{1}{16 \pi} K_{i j} K^{i j}\right],
$$

where, $W \equiv \alpha u^{0}$. A similar equation can be written [2] for the lapse function.

The shift vector $\beta^{i}$ is further decomposed [21]: $\beta^{i}=$ $G^{i}-(1 / 4) \nabla^{i} \chi$, and introduced into the ADM momentum constraint equation to obtain two elliptic equations

$$
\begin{gathered}
\nabla^{2} \chi=\nabla_{i} G^{i} \\
\nabla^{2} G^{i}=2 \nabla_{j} \ln \left(\alpha \phi^{-6}\right) K^{i j}-16 \pi \alpha \phi^{4} S^{i} .
\end{gathered}
$$

These equations have been solved with boundary conditions provided by the first terms in a multipole expansion of the fields as described in [12]. An equation for the extrinsic curvature $K^{i j}$ follows [2] from the ADM momentum constraint equation with the maximal slicing condition.

This set of equations (2), (6)-(9) is solved numerically using an iterative algorithm based upon a specially designed elliptic solver. This method consists of a combination of multigrid algorithms and domain decomposition techniques 12, 23 and utilizes a code which was developed independently of the hydrodynamics code of [1,2].

Solutions are obtained for specific values of the coordinate distance between stars and the total baryonic mass. In this way we can construct a constant baryonic-mass sequence of orbits with a minimum number of code runs. This sequence is a collection of semi-stable orbits that are connected by the inspiral motion of the stars. 
The problem consists essentially in the numerical solution of a set of elliptic equations. The cases of corotating and irrotational systems share the same set of equations for the metric fields. The irrotational systems, however, demand the solution of an extra elliptic equation (2) for the description of the stellar internal structure. This poses a very special problem since the boundary condition (5) is to be satisfied on the stellar surface and not on the grid boundaries as for the rest of the elliptic equations. This is challenging to implement numerically on a fixed Eulerian grid, since the stellar surface is a spheroid embedded in a Cartesian grid. We refer the reader to 23] for the details of our approach to this calculation. Note, however, that this is a completely different numerical approach to that of Bonazzola et al. who utilized spectral methods to solve the elliptic equations.

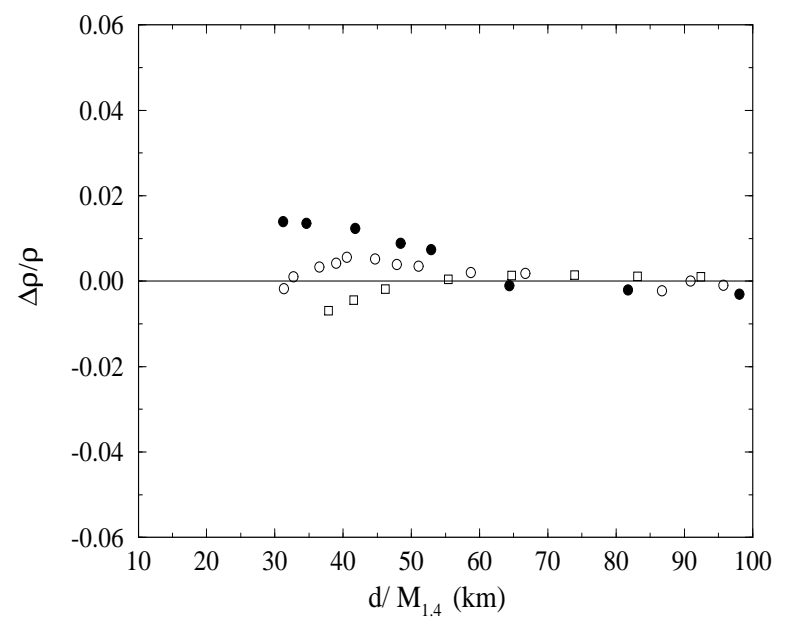

FIG. 1. Change in central density relative to a single isolated star $(\Delta \rho / \rho)$ as a function of the coordinate distance. The black (white) circles represent our calculations for sequences with $\left(M_{G} / R\right)_{\infty}=0.19(0.14)$. The squares show the result obtained by Bonazzola et al. for a sequence composed of stars with $\left(M_{G} / R\right)_{\infty}=0.14$.

To quantify the numerical accuracy, the code was tested against the Newtonian irrotational sequences obtained by Uryū and Eriguchi [24] in two different ways. In one test, the code was stripped of all relativistic terms to reduce it to Newtonian physics. In a second test, orbits for low mass stars $\left(M_{B}=0.10 M_{\odot}\right)$ were calculated using the relativistic code to approach the Newtonian regime $\left(M_{G} / R \approx 0.01\right)$. Comparisons were done for orbits with fixed separation distances between stellar centers $(\tilde{d}$ in the units of [24]). Most importantly, both of these tests exhibit the Newtonian expectation of no discernible change in central density relative to that of isolated stars, with $(\Delta \rho / \rho \lesssim 0.1 \%)$. The resulting values (and percentage difference from results of [24]) for total energy, total angular momentum, and orbital angular frequency were as follows: For the first test, $\tilde{d}=3.53$, and $\tilde{E}=-1.142(0.1 \%), \tilde{J}=1.278(4 \%)$, and $\tilde{\Omega}=0.241(3 \%)$; In the second test, $\tilde{d}=3.88$, and $\tilde{E}=-1.082(4 \%), \tilde{J}=1.413(1 \%)$, and $\tilde{\Omega}=0.203(6 \%)$. These results are consistent with the expected numerical accuracy of this comparison. The code was also tested simulating corotating binary orbits (see 12).

Finally, Flanagan 22] pointed out an inconsistency in the definition of momentum density in the original hydrodynamics calculations [1] 3 . This problem is not present in these calculations.

\section{RESULTS}

For the present study we have found solutions to the initial value equations described above for semi-stable circular orbits for a binary system of identical neutron stars extending from the post-Newtonian regime to the innermost orbit for which we obtain a stable solution.

Figure 11 shows the fractional change $\Delta \rho / \rho$ in the proper central rest-mass density relative to the central density $\rho_{\infty}$ of an isolated star of the same resolution. Results are plotted as a function of the normalized coordinate distance; i. e. $d / M_{1.4} \equiv d /\left(M_{G} / 1.4 M_{\odot}\right)$.

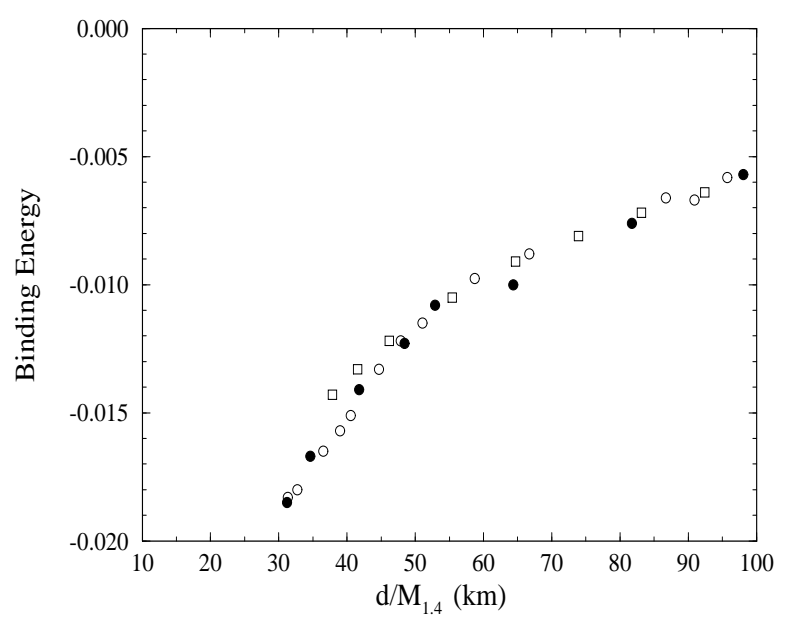

FIG. 2. Binding energy defined as $\left(M_{G}-M_{G \infty}\right) / M_{G \infty}$, where $M_{G}$ is half the ADM mass of the system, vs coordinate distance. The black (white) circles correspond to the sequence of stable orbits with $\left(M_{G} / R\right)_{\infty}=0.19(0.14)$. The squares show the result obtained by Bonazzola et al. for a sequence composed of stars with $\left(M_{G} / R\right)_{\infty}=0.14$.

The squares show the orbits obtained by Bonazzola et al. 11 for a system composed of stars with $\left(M_{G} / R\right)_{\infty}=$ 0.14 . The black (white) circles represent our calculations for sequences with $\left(M_{G} / R\right)_{\infty}=0.19(0.14)$. The numerical error in $\Delta \rho / \rho$ for our irrotational points is conservatively estimated to be $\sim \pm 0.005$ based upon the code tests here described as well as various convergence tests.

Note, that both the Bonazzola et al. results and ours for $\left(M_{G} / R\right)_{\infty}=0.14$ show only a very slight increase 
in the central density before the tidal effects begin to dominate at short separations. The difference between the two curves is probably attributable to differences in the numerical algorithms applied (i.e. coordinate mesh, boundary conditions, elliptic solver, etc). Nevertheless they are consistent to within numerical accuracy. We emphasize that our point is not about quantitative details at the level of \pm 0.005 in $\Delta \rho / \rho$, but about the qualitative trend of increasing central density as the compaction ratio increases.

The sequence with $\left(M_{G} / R\right)_{\infty}=0.19$, clearly, raises above the zero line, ending in a maximum value of $\sim 1.5 \%$ increase in the central density for the last stable orbit found. This result agrees with a similar trend of increasing compression with increasing compaction ratio, presented recently by Bonazzola et al. [25]. This trend derives from the fact that the compression effect competes with the tidal deformation and the latter is stronger for more extended (i.e. smaller compaction ratio) stars.

In both cases the central density approaches the isolated-star limit at large orbital separations. We have checked that the same trend emerges in a plot of the average density, so this effect could not be an artifact of the stellar center being a special point.

As a final point, figure 2 shows the relative binding energy of the system defined as $\left(M_{G}-M_{G \infty}\right) / M_{G \infty}$, where $M_{G}$ is half the ADM mass of the system. We note that the relative binding energy does not depend strongly on the compaction ratio within the numerical accuracy of our results. Another feature of these curves is that they lack a turning point in the sequence. This is in qualitative agreement with the Newtonian irrotational sequences for polytropic index $n=1$ obtained by Uryu and Eriguchi 24.

In summary, the present independent study has obtained the qualitative result of increasing central density as an irrotational binary orbit decays, for neutron stars with compaction ratio of 0.19 . The magnitude of the effect, however, is significantly less than that of the previously reported hydrodynamic results [1 3 . Preliminary recent results [26] indicate that such reduction in compression effect is consistent with the effect of a correction proposed by Flanagan [22] when applied to the hydrodynamic simulations.

Although it is evident that some compression effect exists, it is not yet clear whether this remaining effect is real or a consequence of the conformally flat metric approximation. The magnitude of the apparent effect noted here is roughly comparable to the possible uncertainty introduced by this approximation (cf. [4]). The ultimate test of whether the effect is real will therefore require an accurate fully dynamical relativistic treatment. Such a test is hopefully coming from the neutron-star Grand Challenge collaboration.

Work at University of Notre Dame supported by NSF grant PHY-97-22086. Work at the Lawrence Livermore
National Laboratory performed in part under the auspices of the U. S. Department of Energy under contract W-7405-ENG-48 and NSF grant PHY-9401636.

[1] J.R. Wilson and G.J. Mathews, Phys. Rev. Lett. 75, 4161 (1995).

[2] J.R. Wilson, G.J. Mathews, and P. Marronetti, Phys. Rev. D54, 1317 (1996).

[3] G. J. Mathews and J. R. Wilson, Astrophys. J. 482, 929 (1997).

[4] G. J. Mathews, P. Marronetti and J. R. Wilson, Phys. Rev. D58, 043003 (1998).

[5] D. Lai, Phys. Rev. Lett. 76, 4878 (1996).

[6] See [4] and references therein.

[7] E. Flanagan, Phys. Rev. D58, 124030 (1998).

[8] K. Thorne, Phys. Rev. D58, 124031 (1998).

[9] T. W. Baumgarte, G. B. Cook, M. A. Scheel, S. L. Shapiro, and S. A. Teukolsky, Phys. Rev. Lett. 79, 1182 (1997); Phys. Rev. D 57, 6181 (1998), Phys. Rev. D 57, 7299 (1998).

[10] M. Shibata, T. W. Baumgarte, and S. L. Shapiro, Phys. Rev. D58 (1998) 023002.

[11] S. Bonazzola, E. Gourgoulhon, and J.-A. Marck, Phys. Rev. Lett. 82, 892 (1999).

[12] P. Marronetti, G. J. Mathews, and J. R. Wilson, Phys. Rev. D58 (1998) 042822.

[13] L. Bildsten and C. Cutler, Astrophys. J. 400, 175 (1992).

[14] F. A. Rasio, and S. L. Shapiro, Class. \& Quantum Grav., in press, section 5.2, (gr-qc/9902019).

[15] S. Bonazzola, E. Gourgoulhon, and J.-A. Marck, Phys. Rev. D56, 7740 (1997).

[16] S. A. Teukolsky, Astrophys. J. 504, 442 (1998).

[17] M. Shibata, Phys. Rev. D58 (1998) 024012.

[18] H. Asada, Phys. Rev. D57, 7292 (1998).

[19] E. Gourgoulhon, Phys. Rev. D, submitted (1998), grqc/9804054).

[20] J. W. York, Jr., in Sources of Gravitational Radiation, edited by L . Smarr (Cambridge Univ. Press, Cambridge, England, 1979), p. 83.

[21] J. M. Bowen and J. W. York Jr., Phys. Rev. D21, 2047 (1980).

[22] E. Flanagan, Phys. Rev. Lett. 82, 1354 (1999).

[23] P. Marronetti, G. J. Mathews, and J. R. Wilson, Proceedings of the XIX Texas Symposium in Cosmology and Relativistic Astrophysics, in press, gr-qc/9903105.

[24] K. Uryū and Y. Eriguchi, Mon. Not. Roy. Astron. Soc. 296 (1998) L1.

[25] S. Bonazzola, E. Gourgoulhon, and J.-A. Marck, Proceedings of the XIX Texas Symposium in Cosmology and Relativistic Astrophysics, in press, gr-qc/9904040.

[26] G. J. Mathews, J. R. Wilson, and C. Blakelock, Proceedings of the XIX Texas Symposium in Cosmology and Relativistic Astrophysics, in press. 